Voix et Images

volxetimages

\title{
Madeleine Gagnon : Les constantes d'une écriture...
}

\section{Madeleine Boulanger}

Volume 8, numéro 1, automne 1982

Madeleine Gagnon

URI : https://id.erudit.org/iderudit/200364ar

DOI : https://doi.org/10.7202/200364ar

Aller au sommaire du numéro

Éditeur(s)

Les Presses de l'Université du Québec

ISSN

0318-9201 (imprimé)

1705-933X (numérique)

Découvrir la revue

Citer cet article

Boulanger, M. (1982). Madeleine Gagnon : Les constantes d'une écriture... Voix et Images, 8(1), 45-51. https://doi.org/10.7202/200364ar d'utilisation que vous pouvez consulter en ligne.

https://apropos.erudit.org/fr/usagers/politique-dutilisation/ 


\title{
Madeleine Gagnon: Les constantes d'une écriture...
}

\author{
par Madeleine Boulanger, Université Laval
}

Depuis plus de douze ans, Madeleine Gagnon trace un itinéraire assez fidèle à lui-même. Des Morts-Vivants (1969) jusqu'Au Cœur de la lettre (1981), elle nous entraîne à sa suite sur la piste des origines de la vie et de l'écriture. Des souvenirs d'enfance, vus à travers la forêt, le feu, l'eau de la rivière, le train qui serpente dans la Vallée et la mort qui rôde, elle réinvente une archéologie inversée où, au lieu de fossiliser, on refait la vie et l'écriture, dans leurs fibres premières.

Entre ces points-limites, il y aura eu réflexion et autocensure dans Retailles et la Venue à l'écriture; œuvre de combat et de dénonciation, Pour les femmes et tous les autres; étape nécessaire et combien riche du délire verbal, Portraits du voyage et Poélitique; à nouveau poésie et recherche du langage, mais cette fois en leur manque, Antre, jusqu'au retour de la grandmère et la redécouverte des origines, Lueur. L'auteur aura franchi - à travers un discours féminin authentique - toutes les étapes du combat jusqu'à la tendresse.

Nous examinerons les éléments constants de cette écriture: ce que l'auteur elle-même en a dit et pensé.

D'abord Madeleine Gagnon ne croit pas aux Muses. Selon elle, qui veut peut écrire. Il faut s'entendre: qui désire écrire en a déjà quelque possibilité. L'être profond a déjà sa pente, son «éprouvé vital (...) le cuisant désir de créer s'épanche par les lignes de force de l'être lesquelles sont ramassées, orientées, chargées par les conditionnements." 1

Et le désir conduit au plaisir créateur, non au geste paresseux qui nous serait donné. Il faut plonger. "Nous savons que nous n'écrivons que lorsque le saut est accompli, mais pour l'accomplir, il faut d'abord écrire, écrire sans fin... ${ }^{2}$ II faut aussi un certain goût et certaines aptitudes naturelles. «Celui qui n'a pas de grande appétence pour lire et qui n'a pas, dès sa jeunesse, dévoré les livres n'aura pas le désir d'écrire...» ${ }^{3}$ Le recueil de dix nouvelles, Les Morts-Vivants, est né de ce désir.

Dans le même ordre d'idée, l'écriture est un geste organique tout autant que cérébral. L'écriture: chair et sang qui circule de partout. Les forces les 
plus vives de l'être précèdent et accompagnent l'écriture; elles en sont le substrat, l'humus, sans quoi rien n'est possible. Elles tissent ce que sera le style et s'appellent élans vital, mental, verbal qui alimenteront la flamme: avoir de la peine, éprouver, jalouser, craindre, aimer, désirer, jouir, etc. Architecture qui participe de nos gènes, de nos tissus, de nos membres, de nos articulations, tout autant que du matériau intellectuel/cérébral. Conjointement à Hélène Cixous et Annie Leclerc, Madeleine Gagnon nous raconte longuement dans la Venue à l'écriture ${ }^{4}$ cette aventure appelée Mon corps dans l'écriture et la rapproche de cette autre grande aventure de l'accouchement. «Si l'écriture relève de tout l'être et en suscite la renaissance, c'est qu'elle se veut une tentative, un moyen de ne pas mourir, du moins de se débattre entre l'instinct de conservation et l'instinct de mort. ${ }^{5}$ Quoi de plus somatique que cette lutte à finir? Et si la mort, malgré tout, a commencé son œuvre, l'écriture peut régénérer les tissus à la façon de la vision d’Ezéchiel 6 , ou encore comme on le voit avec les deux ressuscités des Morts-Vivants: Wilfrid et Grégoire?

On ne saurait dépeindre l'œuvre de Madeleine Gagnon sans réfléchir sur un des grands phénomènes de notre temps: l'écriture féminine/féministe. L'auteur de Pour les femmes et tous les autres annonce par ce seul titre la tendance spécifique de ses propos. Certes, comme la plupart de ses contemporaines, elle dénonce tous les pouvoirs y compris le pouvoir phallocrate. Mais elle a soin d'ajouter que «le féminisme n'est pas la guerre des sexes; il la termine». 8 II faudra cependant, pour arriver à cette paix négociée réussir un certain nombre de combats. Se délivrer de sa naissance, de son enfance et du nom du Père. Se libérer de l'éducation/domination. Dénoncer toutes les oppressions socio-économiques et politiques. Ces enjeux ne sont pas sans risques: renversement de pouvoir (?) dans l'engagement politique ou féministe. ou du moins enfermement narcissique où, après avoir été marginale, la situation des femmes deviendrait parallèle. Les siècles de silence ont fait place à une abondance de parole/écriture: flot qu'on ne pourra plus endiguer. Les femmes veulent prendre leur place, toute leur place et l'occuper, dans un certain pouvoir partagé fait d'échange et de prise en charge mutuels. Cette étape franchie, il y aura place pour la tendresse et l'amour, où le plaisir répond au désir, jusqu'au discours féminin gratuit. Écrire pour le plaisir d'écrire... écrire parce qu'on est heureuse et qu'on veut le dire...

L'écriture n'est pas que le texte mais aussi l'avant (gestation) et l'après (influence). Dans une démarche autobiographique très fréquente, l'auteur utilise toute cette moisson cueillie depuis l'enfance:

La mémoire est un gros sac rempli de brouillons et de griffonnages, sans enveloppes, ni adresse, ni timbres, avec parfois des sceaux indistincts, de vagues signatures ou dates, où l'on peut piger à pleines mains. La cueillette se fait souvent en rêve et au matin, quand la conscience se reprend en main, reprend pied, elle essaie de retracer son chemin clair au milieu d'un double fouillis, celui des souvenirs obscurs et celui du rêve illogique. ${ }^{9}$ 
Dans Lueur ${ }^{10}$ on retrouvera ces matériaux admirablement agencés. L'auteur rend un hommage vibrant à la parole, à l'écriture, à ces mots qui se dégagent avec tant de mélodie. Musique, tableau, fête, triomphe, c'est le deuxième acte de ce geste créateur, "qui sur un coin de la page blanche découvre le texte, le fait apparaître, émerger du silence ${ }^{11}$ comme la pierre dégage petit à petit la statue qu'elle cachait. Les retombées de l'œuvre et son influence constituent le troisième temps de ce même geste. Pour l'auteur de Retailles, tout cela est du texte et les trois mouvements, quoique distincts, sont inséparables. Comme l'indique ce titre, il n'y a qu'à recoudre toutes ces pièces et l'œuvre est née.

Cette influence est orchestrée par le lecteur et le critique. À l'instar de plusieurs contemporains, Madeleine Gagnon veut se donner, la première, ce double rôle. L'auto-censure "est un droit et peut-être un devoir pour un auteur.»12 Qui mieux que lui peut savoir et comprendre ce que contient son texte? "Lui seul connaît les choses vivantes derrière son écriture." 13 II peut même reconnaître l'aspect négatif de ce qu'il a produit. S'il est sincère et objectif, sa parole en vaut bien d'autres. Son honnêteté intellectuelle en matière d'auto-critique ajoutera même du prix et de la crédibilité à son œuvre. Madeleine Gagnon se fait aussi critique de la critique. L'écrivain se livre à tous les publics: ses détracteurs et ses défenseurs. Ses indifférents aussi. Sans être meurtrie ou offusquée outre mesure, elle se montre quand même surprise et étonnée. Sans être une mordue de l'efficacité, Madeleine Gagnon ne peut rester indifférente à une certaine critique acerbe. Dans Lueur, elle s'invente (?) un interlocuteur, qui, pour ne pas être. nommé, n'en est pas moins vraisemblable. Voilà une démonstration qui pourrait être endossée par les media de tout acabit... Accusation triviale où la gratuité est condamnée d'avance, au profit de la rentabilité, par la catégorisation rassurante et stérile. L'intervenant invisible joue même à se montrer généreux et prêt aux concessions... II accepterait l'anti-roman et la lutte des femmes. Est-il féministe? ou oppresseur? La finale fait rêver. Cette démonstration par l'absurde prépare à l'idée de la destruction réelle (?) abondamment décrite... ${ }^{14}$ Et ce long paragraphe se termine sur l'image du fer rouge. Jour de la colère où on refuse la ferrade... On ne marque que les bêtes et les esclaves. Drame de la brisure corporelle, intimement liée à la destruction d'une écriture, double courant d'existence. On croirait entendre Nounouche expliquer à Luchie les décortications professorales... ${ }^{15}$ Heureusement, il y a une autre critique, douée d'imagination, d'enthousiasme, de fraîcheur admirative, en un mot d'un regard neuf, toutes qualités qui relèvent de l'affectif et du cérébral conjugés. Ressemblance, connivence et sympathie où auteur-lecteur-critique ne font plus qu'un.

L'auto-censure authentique est rendue possible grâce à l'imbrication constante de la théorie et de la fiction, l'une complétant l'autre de façon assez heureuse. Pouvoir aller de l'une à l'autre sans heurt ni soubresauts «utilisant pour cela, toutes sortes d'écritures... ${ }^{16}$ Réalisme constructeur qui peut traverser les plus belles pages et nous conserver une lucidité qui n'exclut ni le ludique ni l'onirique. "Imaginer une fiction est une façon de s'ouvrir une nouvelle voie dans la réalité et par là d'intervenir sur elle et de la modifier.»17 
On atteint tout autant la vérité par l'art que par la science... Hugues Corriveau a reconnu cette notion dans Antre:

En joignant dans ses textes le fictif au théorique avec un acharnement qui déroute, elle produit une nouvelle forme du discours. Elle cherche la fracture d'unité, la brisure de la linéarité confondante du texte classique, dans une tentative de faire circuler les sens multiples et parfois éclatés. ${ }^{18}$

Porte ouverte sur le grand large où tous les possibles se rencontrent. Madeleine Gagnon s'en explique à Jean Royer, à propos de Lueur:

II y a aussi dans Lueur une certaine organicité de la théorie et de la fiction. Je ne sais pas si j'y suis arrivée. Mais ce désir de travailler organiquement la théorie et la fiction c'est celui de ne pas être dans l'une contre l'autre. Être dans l'une et non dans l'autre, c'est une censure des deux. On m'a souvent reproché ce collage du théorique qui traverse le poétique mais ces critiques ne m'ont pas tellement dérangée. II ne faut pas oublier que toute théorie est construite sur du délire et qu'en même temps toute écriture de fiction est fondée sur de la théorie, sur des parcelles de pensée, de philosophie... Une barrière entre les deux empêche, chacune dans leur champ, ces recherches de progresser. ${ }^{19}$

Se consacrer à l'écriture c'est aussi se donner à la parole et combattre avec le langage. La parole, les paroles "enfin signifiantes. "20 Dès Les MortsVivants, Madeleine Gagnon se disait artisane des mots:

J'aime les mots qui s'accumulent. J'aime les tas de mots qui s'entassent [sic] quand toute la maisonnée ronfle et que la fournaise en bas bougonne d'être seule à ne pas dormir. J'aime les mots qui s'additionnent sous le soleil...21

Beauté des mots et des phrases jusqu'à l'éblouissement: “Je me tenais prise de vertige, les mains tremblantes sur le garde-fou du langage. "22 Ouvrière du langage, l'auteur ira même Au cour de la lettre (1981) pour en goûter toute la saveur et la variété. Humble devant les mots qui sont ses maîtres, elle n'hésite pas à utiliser le registre complet du langage, y voyant de nombreux éléments de création. Pierre Nepveu décrit ce foisonnement verbal:

Il y a toujours eu dans l'écriture de Madeleine Gagnon une cohabitation de divers niveaux ou types de langage. Dans un recueil comme Pour les femmes et tous les autres, cette diversité tenait du collage: citations, voix de la femme «ordinaire», flot poétique mêlant le joual, les images les plus crues et les références théoriques à Marx, Freud, Beauvoir, etc. (...) cette discontinuité n'existe qu'en surface: elle est celle d'une écriture qui cherche à varier les points de vue (...) comme toute écriture digne de ce nom. ${ }^{23}$

La lutte reste ouverte: écrire, c'est combattre avec les mots, avec le langage, dans une recherche constante aboutissant souvent au délire verbal plus ou moins dirigé ${ }^{24}$. Des résistances externes voudront parfois réduire le flot incessant et à son tour l'abondance de paroles pourra sembler agresser: " $\mathrm{Ce}$ qu'autour ils nommèrent délire m'apprit le danger du silence.» ${ }^{25}$ Réaména- 
gements internes (lecture-critique-théorie-fiction), vocabulaire de l'écorchure, de l'immolation (dénonciation de tous les pouvoirs-répressions), langage percutant et vrai, propre à toutes les luttes comme à toutes les jouiissances.

Une expression revient souvent sous la plume de Madeleine Gagnon, expression qui n'est pas sans lien avec les trois premières constantes énoncées. Si on ne croit pas aux Muses, si le travail de l'écriture est aussi organique que cérébral et - en l'occurrence - spécifiquement féminin, on peut facilement s'appeler «artisane» des mots. À lui seul, le titre Retailles en serait une excellente démonstration. En effet, le métier d'écrivain ressemble étrangement au travail artisanal. Remplacer nos courtes vues par nos courtepointes et «découdre jusque dans ses replis cachés des siècles de domination. ${ }^{26}$ Ouvrage de Pénélope rappelant aussi l'infinie patience de nos aieules et leur silence millénaire...

Retournons voir aussi en notre histoire écrite. Soulevons ce coin de catalogne. Dédoublons. Décentrons. Ou bien sous le poil d'ours, c'est dentelle. C'est satin. Faufilons toutes nos retailles. Reconstituons les archives. Ecritures en train de s'écrire. Paroles en train de se jouer. C'est I'histoire que nous tramons. ${ }^{27}$

L'écriture, un artisanat d'un genre particulier. Geste de confiance et d'espérance, «travail de taupe» ${ }^{28}$, qui bâtit l'envers du canevas. Pour Madeleine Gagnon, le champ de la création "constitue l'histoire à l'envers telle que nous l'avons toujours vécue." ${ }^{29}$ Pour réussir l'endroit, il faut connaître l'envers et travailler «l'autre côté, le dissemblable, le dedans, l'obscur, la nuit océan.» ${ }^{30}$ "Doublure de soie d'une tapisserie géante dont l'endroit nous échappe»31, l'cuvre joue le jeu du miroir et du reflet: "Se voir à l'envers et mieux se reconnaître.» 32 Une seule récompense anticipée: "Les courtepointes de nos grandmères réservaient toujours des surprises. " ${ }^{33}$ Récompense la plus appréciée.

L'écrivain va plus loin encore et appréhende le vide pour le combler. Procédé génétique de la vie et de l'écriture étroitement liées. Antre en est la meilleure expression et Lueur poursuit le processus jusqu'au retour de la grandmère et des origines perdues. "Nous sommes produits des brèches qu'une longue et patiente conscience a creusées. Fissures, entre tous ces pouvoirs opaques. ${ }^{34}$ Le vide: chemin que se trace l'œuvre et par lequel elle passera: «... I'on n'écrit que si l'on atteint cet instant vers lequel l'on ne peut toutefois se porter que dans l'espace ouvert par le mouvement d'écrire. " ${ }^{35}$ C'est la recherche des blancs, des omissions, des mensonges à combler. Cette démarche déjà annoncée dans $L a$ Venue à l'écriture ${ }^{36}$ est plus précise encore dans Antre: « J'écris pour raconter les temps et les espaces entre les riens, les lieux entre les trous, interstices d'où l'on aurait bien pu ne jamais revenir et n'en jamais parler. „37 Vide et manque qui seront comblés jusqu'à la plénitude...

Dès les premières pages des Morts-Vivants, et comme le titre le dit sans équivoque, LA PENSEEE DE LA MORT A PROBABLEMENT ÉTÉ LE PREMIER DÉCLENCHEUR DE L'ÉCRITURE DE MADELEINE GAGNON. On écrit pour conjurer la mort, sa mort et toutes les mots: je/tu/nous. Mais il faut compter aussi avec la vie qui reprend sans cesse ses droits, de sorte qu'il y a dans 
l'écriture un jeu constant entre Eros et Thanatos, sœurs ennemies qui veulent régner à tour de rôle. Mouvement ambivalent de toute existence: celle de la vie comme celle de l'écriture. "Verrai-je la mort sortir du cratère et n'être plus désormais qu'un long palpitement du souvenir de la mort dans la vie?" 38 Philipa ${ }^{39}$ a longtemps pleuré sur sa laideur et s'est interrogée sur la mort de la belle Hélène de Troie. Stéphanie ${ }^{40}$ a longuement réfléchi sur le sort de François qui avait choisi de s'arrêter en route. Mais c'est Zoé ${ }^{41}$ qui triomphe, les bras chargés de fleurs...

Philipa-Stéphanie-Zoé: la même petite fille devenue femme douce, tendre et obstinée, parmi tant d'autres Québécoises... Merveilleux spectacle de femmes réunies dans le partage de leur écriture et de leur joie. Des femmes devenues amies par delà les barrières des origines et des concepts... ${ }^{42}$

\section{QUE LA VIE CIRCULE ET QUE SE POURSUIVE L'ÉCRITURE FÉMININE...}

1. Pierre Debray-Ritzen, Psychologie de la littérature et de la creation litteraire. Paris, Retz, La Bibliothèque du CEPL, p. 158.

2. Maurice Blanchot, Le Livre à venir. Paris, Gallimard, c1959, p. 306.

3. Pierre Debray-Ritzen, op. cit., p. 40.

4. 2e partie: Mon corps dans l'écriture, pp. 63-116.

5. Pierre Debray-Ritzen, op. cit., p. 47.

6. Ezéchiel 37, 1-14.

7. pp. $21-46$ et $67-79$.

8. Liberté, Année 1976. Numéro 106-107. Vol. 8, n 4-5. Juillet-octobre, p. 252.

9. Les Morts-Vivants, Entre deux trains: pp. 140-141.

10. pp. 137-138.

11. Raymond Jean, Actes du Colloque de Strasbourg, présentés par Michel Mansuy, Paris, Ed. Klincksieck, c1971, p. 131p.

12. Pierre Debray-Ritzen, op. cit., 134p.

13. Ibid., p. 213.

14. pp. 51-52; pp. 144-145.

15. Les Morts-Vivants, Luchie et Nounouche, pp. 85-86.

16. La Venue à l'écriture, p. 83.

17. Ibid., p. 103, citant Eva Forest, Journal et Lettres de prison.

18. Le Devoir. Le plaisir efficace jusqu'a l'Antre de Madeleine Gagnon. 30 décembre 1978, p. 11.

19. Le Devoir. Madeleine Gagnon. Explorer les premieres traces du langage. 26 mai 1979, p. 17.

20. Antre, p. 41.

21. p. 161.

22. Le Devoir. 26 mai 1979, p. 17, op. cit.

23. Lettres québécoises, $n^{\circ} 12$, novembre 1978, pp. 15-16. L'Antre et la Sorcière: Madeleine Gagnon et Francine Déry.

24. Portraits du voyage...

25. Antre, p. 41.

26. p. 51.

27. La Venue à l'écriture, pp. 105-106.

28. Madeleine Gagnon, Chroniques, vol. 1, $n^{\circ} 6 / 7$, juin-juillet 1975. p. 53. 
29. La Venue à l'écriture, p. 103.

30. Ibid., p. 104.

31. Lueur, p. 142.

32. Portraits du voyage, p. 42.

33. Claudette Tougas. La Presse, 25 juin 1977. Retailles de deux femmes qui parlent.

34. Lueur, p. 41

35. Maurice Blanchot, L'Espace littéraire, Gallimard, c1955, p. 234.

36. p. 92.

37. Antre, p. 5.

38. Retailles, p. 146.

39. Les Morts-Vivants, La Laide, pp. 9-19.

40. Les Morts-Vivants, Entre deux trains, pp. 135-174.

41. Lueur, p. 113.

42. Au fond des yeux. 25 Québécoises qui écrivent. Nouvelle Optique, c1981. 109p. $21 \mathrm{~cm}$. Photographies de Kéro. 